\title{
Milli-arcsecond Scale Rotation Measure in Two CSSs
}

\author{
F. Mantovani, R. Ricci \\ Istituto di Radioastronomia, Bologna, Italy
}

W. Junor

University of New Mexico, Albuquerque, USA

D.J. Saikia

NCRA, TIFR, Pune, India and Queen's University, Kingston, Canada

C. Salter

Arecibo Observatory, HC3 Box53995, Arecibo, PR 00612, USA

\begin{abstract}
Two Compact Steep-spectrum Sources (CSSs), 0548+165 and 1524-136, have been observed in dual polarization with the VLBA at 4.9 and $8.4 \mathrm{GHz}$. Rotation measures (RMs) up to $10^{4} \mathrm{rad} \mathrm{m}^{-2}$ were derived for the jets of both sources. These high values possibly arise due to the external medium interacting with the radio jets, with measured depolarization being due to small-scale inhomogeneities in the medium.
\end{abstract}

Subarcsec polarimetric observations of CSSs have often provided evidence in favour of interaction of their jets with dense clouds of gas, as for example in the case of 3C147 (Junor et al. 1999). Two sources, 0548+165 and 1524-136, were selected from a list of CSSs with reasonable degrees of polarized emission, polarization percentages that decrease with frequency, and values of $\mathrm{RM}>400 \mathrm{rad}$ $\mathrm{m}^{-2}$ (Mantovani et al. 1994), for further dual-polarization VLBA observations at 4.9 and $8.4 \mathrm{GHz}$.

The observations were done at five frequencies, $8405 \mathrm{MHz}, 5094 \mathrm{MHz}, 4852$ $\mathrm{MHz}, 4657 \mathrm{MHz}, 4619 \mathrm{MHz}$. The images, convolved with a two-dimensional circular-symmetric Gaussian beam 6 mas in width, allowed us to determine RM values in several highly polarized regions along the jets of the two CSSs. Values of $>4000 \mathrm{rad} \mathrm{m}^{-2}\left(\delta(\theta) \simeq 570^{\circ}\right)$ and up to $10^{4} \mathrm{rad} \mathrm{m}^{-2}\left(\delta(\theta) \simeq 1420^{\circ}\right)$ were found for $0548+165$ and $1524-136$ respectively. Such large RMs are likely to be due to dense ionised regions in the external medium, which could be due to material swept up by the radio source, the line-emitting regions, or infalling gas possibly fueling the radio source.

In $0548+165$ (Fig. 1) the three regions $\mathrm{A}, \mathrm{B}$, and $\mathrm{C}$, all show large values of RM, much larger than the RM of $\leq 100 \mathrm{rad} \mathrm{m}^{-2}$ produced by the ISM of the Galaxy. The largest value of RM is found in region $\mathrm{C}$, where the jet starts bending. The RM then decreases as one moves farther out along the jet through 


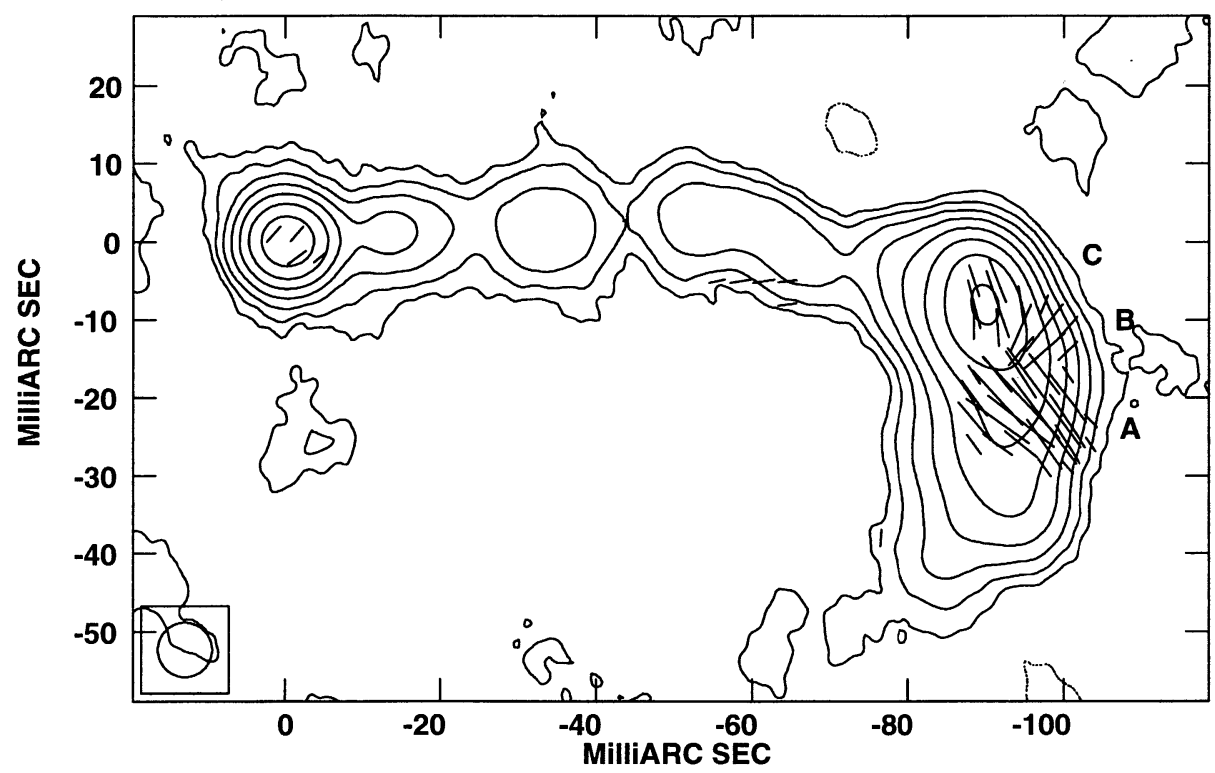

Figure 1. VLBA+Y1 image of $0548+165$ at $4619 \mathrm{MHz}$ convolved with a 6 mas beam. Contours are $-1,1,2,4,8,16,32,64,128 \mathrm{mJy} /$ beam. The peak flux density is 142.9 $\mathrm{mJy} /$ beam. Projected E vectors are plotted. A vector of 1 mas $=0.2 \mathrm{mJy} / \mathrm{beam}$.

region $\mathrm{B}$ to region $\mathrm{A}$. In the bent region, where the jet clearly interacts strongly with the external medium, the depolarization is $\mathrm{p}_{4.6} / \mathrm{p}_{8.4}=0.26$.

In 1524-136 the RM values change along the jet. The peak of polarized emission is found towards the end of the jet, just after a region of lower RM, where the jet changes direction. The jet components also depolarize. Integrating the total and polarized flux density of the whole source, the depolarization value is $\mathrm{p}_{4.6} / \mathrm{p}_{8.4}=0.28$.

Estimates of the line-of-sight component of the magnetic field in the external screen are possible assuming an 'a priori' electron density, $n_{e} \simeq 0.1-1 \mathrm{~cm}^{-3}$, and a slab thickness of $1 \mathrm{kpc}$. The values obtained for $1524-136$ range up to $100 \mu \mathrm{Gauss}$, and are larger than those for $0548+165$, which are in the range 1.5 - $15 \mu$ Gauss. Since X-ray measurements are not available, and the angle of the jet axis to the line-of-sight is not known for either source, the parameters cannot presently be better constrained.

\section{References}

Junor, W., Salter, C.J., Saikia, D.J. et al. 1999 MNRAS, 308, 955

Mantovani, F., Junor, W., Fanti, R. et al. 1994 A\&A, 292, 59 